\title{
Evolution de l'habitat rural dans le Jura central suisse (Canton de Neuchâtel)
}

\author{
Frédéric Chiffelle
}

L'habitat rural de la région étudiée (Jura central suisse) subit actuellement des modifications qui affectent à la fois la conception architecturale de la maison rurale elle-même et le mode de répartition des fermes.

\section{Modifications de la conception architecturale de la maison rurale du Jura central suisse}

Les modifications que nous analyserons sont une illustration de la pensée du géographe français Demangeon que nous voudrions placer en exergue:

"La personnalité foncière de l'habitation rurale ne se compose pas de ces éléments qui changent et qui passent; elle émane surtout de l'ordonnance interne des bâtiments qui est née de besoins agricoles. La maison du paysan donne la solution d'un problème vital qui est de savoir comment s'établiront les rapports réciproques des hommes, des bêtes et des biens.»

Demangeon A., Problèmes de géographie humaine, Paris, Colin, 1952, p. 266

Divers facteurs influencent la forme du principal outil du paysan qu'est la maison rurale: facteurs naturels, économiques, sociaux, techniques et psychologiques. L'incidence de l'économie et de la technique agricoles sur l'architecture rurale apparait actuellement prépondérante. La chaîne jurassienne est traditionnellement comprise dans la zone d'extension de la maison-bloc. On peut recenser deux sous-types de cette maison-bloc dans le Jura central suisse: la maison vigneronne et la maison paysanne.

\section{La maison vigneronne (photo no 1)}

La maison du viticulteur des rives des lacs subjurassiens est une maison-bloc en hauteur selon la terminologie de Demangeon. Le premier étage est entièrement réservé au logement (de même que le deuxième étage là où il existe). Les locaux vinicoles n'occupent guère que le rez-de-chaussée (pressoir et cave à vin) et parfois le sous-sol. Les locaux professionnels prennent ainsi peu de place. Il en découle que les maisons vigneronnes peuvent être con- tiguës sans inconvénients et que cette contiguïté même donne un caractère urbain aux villages viticoles. Il s'ensuit également que la maison vigneronne du Jura suisse est encore sensiblement la même aujourd'hui qu'il y a trois ou quatre siècles: les villages viticoles sont des joyaux d'architecture du XVIe, XVIIe ou du XVIIIe siècle qui ont conservé leur aspect et leur fonction viticoles.

Seuls un accroissement récent des dimensions des domaines viticoles ainsi que des changements de techniques vinicoles ont rendu les anciens locaux trop exigus. La vinification s'effectue de plus en plus dans des caves de grande dimension aménagées spécialement à cet effet (caves collectives ou caves privées). Comme il s'agit de constructions séparées, la maison vigneronne elle-même n'en est que peu modifiée.

\section{La maison paysanne}

La conception de la maison du polyculteur-éleveur est plus étroitement liée à sa fonction agricole que ne l'est celle du viticulteur; elle est "outil» dans une plus forte mesure et comme telle, la maison du paysan matérialise une des adéquations les plus parfaites entre l'architecture et la fonction. Contrairement à la maison vigneronne, elle s'est modifiée de façon si fondamentale depuis trois ou quatre siècles qu'on peut distinguer plusieurs sous-types dans le Jura central suisse; ce sont dans l'ordre chronologique la ferme-pignon, la ferme dite "maltournée» et la ferme dissociée qui est la ferme actuelle.

a. La ferme-pignon (photo no 2) est la ferme du XVIe, XVIIe ou XVIIIe siècle; elle doit son nom à sa façade principale qui est une façade pignon. Le faîte du toit a par conséquent une orientation nordouest/sud-est. Cette ferme est bâtie sur un plan presque carré d'une douzaine de mètres de côté; sa tripartition es! généralement longitudinale, le logement et l'étable étant situés de part et d'autre de la remise. La grange occupe le premier étage.

La ferme-pignon est particulièrement bien adaptée au milieu naturel jurassien. On a volontairement construit la ferme au point de contact entre les fonds de vallée en cultures et le flanc de la montagne en pâturages, concrétisant ainsi la symbiose agriculture-élevage. Cette localisation au point de 


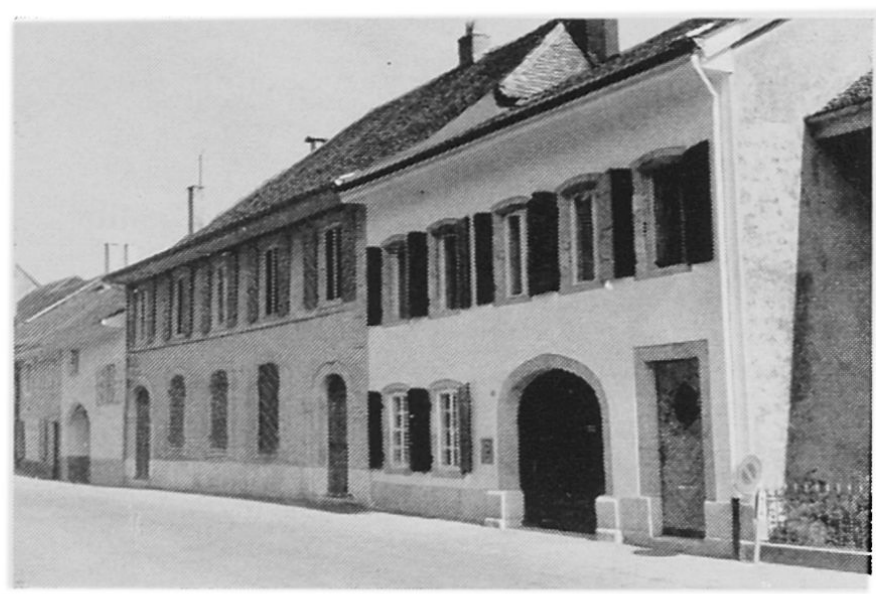

Photo 1. Maisons vigneronnes contiguës à Cortaillod. Locaux professionnels au rez-de-chaussée (porte voûtée donnant sur le pressoir et la cave). Logement au premier étage. Toutes les photos de l'article sont de F. Chiffelle

rupture de pente facilite par ailleurs l'engrangement des récoltes: la ferme étant adossée à la montagne, on accède à la grange par un plan incliné d'une pente relativement faible. Enfin, le toit répond admirablement à la double nécessité d'assurer une bonne isolation thermique en hiver et l'approvisionnement en eau des bêtes et des gens. Rappelons en effet que les températures inférieures à $-10^{\circ}$ ne sont pas rares dans le Jura qui est par ailleurs une région karstique à circulation souterraine des eaux. L'inclinaison favorable des pans du toit permet de retenir une épaisse couche de neige qui sert d'isolation en hiver et qui remplit la citerne au printemps.

La ferme-pignon, parfaitement adaptée à la petite exploitation d'une douzaine de bovins, s'est trouvée trop exiguë pour abriter les récoltes plus abondantes et le bétail plus nombreux issus de la révolution fourragère du XIXe siècle. Accolée à la montagne, la ferme-pignon est difficilement extensible, le faîte de son toit étant perpendiculaire aux courbes de niveau. Pour répondre à ce besoin d'un accroissement du volume de la ferme, les constructeurs ont fait subir une rotation de $90^{\circ}$ au faîte de son toit (photo no 3). La ferme du XIXe siècle a donc été construite selon un axe (marqué par le faîte) parallèle aux courbes de niveau. Elle est une "maisonbloc à éléments transversaux» appelée "maltournée» par les agriculteurs jurassiens qui la comparent à la ferme-pignon classique.

b. La ferme maltournée (photo no 4) a un volume considérable, qui atteint ou dépasse le double de celui de la ferme-pignon: sa base est constituée par un vaste rectangle de quelque trente mètres de longueur et quinze mètres de largeur; la grange a pris des dimensions monumentales puisqu'elle occupe les

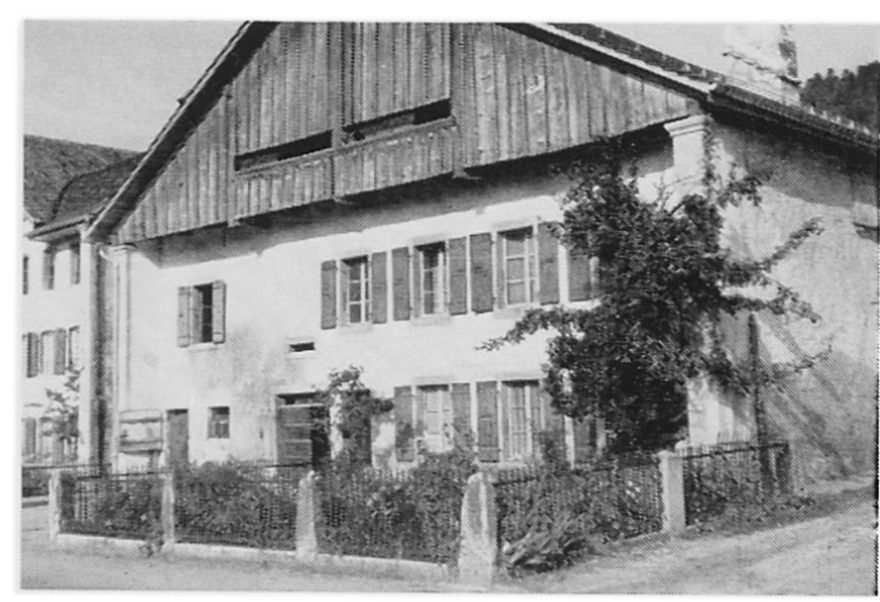

Photo 2. Ferme-pignon de Dombresson à tripartition longitudinale du rez-de-chaussée: étable, fourragère et logement. Brise-vent à chaque extrémité de la façade. Fentes d'aération de la grange aménagées dans la façade-pignon

premier et deuxième étages; l'étable peut accueillir une douzaine de vaches au lieu de six ou huit dans la ferme-pignon. D'un volume appréciable, elle peut encore être allongée sans engagement de frais disproportionnés à cause de son orientation parallèle aux courbes de niveau. Cette grande faculté d'adaptation explique son maintien jusqu'à la Deuxième Guerre mondiale. Depuis 1945, les agriculteurs se plaignent au contraire de son inadaptation; c'est dire que la maison paysanne du Jura suisse traverse actuellement une phase de transition où l'on peut cependant distinguer quelques conceptions majeures: de façon générale, la maison-bloc est abandonnée au profit de ce que nous appellerons la maison dissociée.

c. La maison dissociée, en faveur actuellement, ne constitue pas un retour à la maison-cour que le Jura suisse n'a d'ailleurs jamais connue. Elle résulte de la spécialisation croissante des bâtiments de l'exploitation agricole. Les causes de cet éclatement de la ferme sont multiples: exode agricole, avec l'agrandissement des exploitations et la réduction de la main-d'œuvre qu'il provoque, accroissement des rendements, désir de confort.

Le coût de la construction étant relativement élevé en Suisse, l'agriculteur se contentera le plus souvent d'édifier un ou plusieurs bâtiments annexes tout en conservant l'ancienne ferme.

Le "rural», c'est-à-dire un bâtiment comprenant étable et fenil, est l'adjonction la plus fréquente. Il répond à un accroissement du cheptel (vaches laitières ou bovins d'engraissement). L'élevage et l'engraissement porcins se pratiquent de plus en plus fréquemment dans des bâtiments spécialement conçus à cet effet (photo no 5). L'accroissement de la 


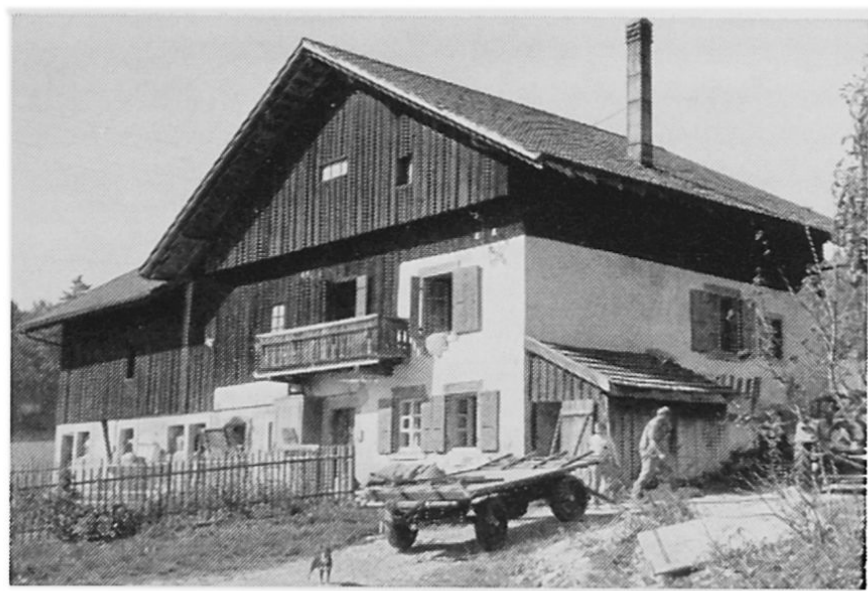

Photo 3. Illustration du passage de la ferme-pignon (bâtiment principal) à la ferme maltournée (étable récente). Ferme des Vieux-Prés sur Chézard

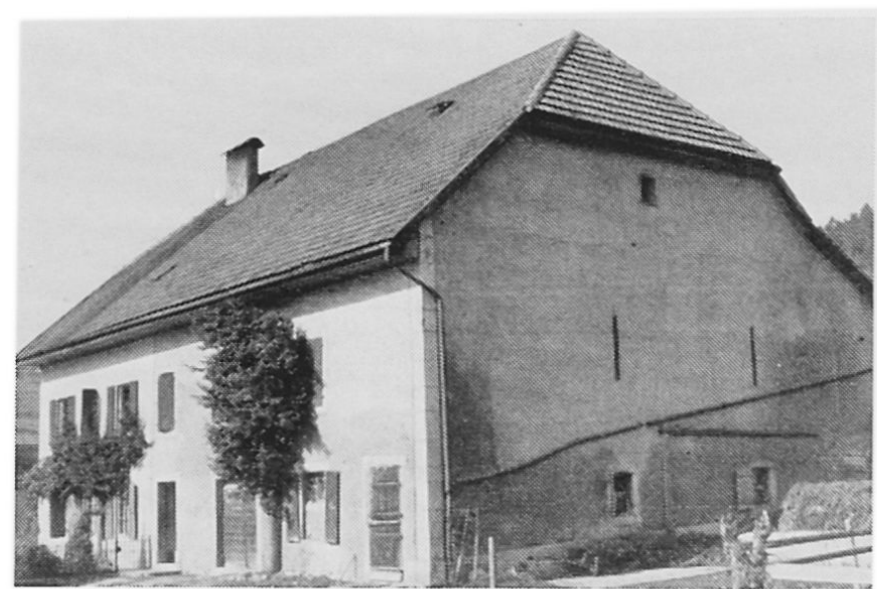

Photo 4. Ferme maltournée de Chézard-St-Martin à tripartition transversale (au rez-de-chaussée: logement, fourragère, étable; au premier étage: logement et grange)

à bâtir, soit, le plus souvent, par des subsides publics, accordés dans le cadre de remembrements parcellaires. Les subsides publics, qui couvrent approximativement les deux tiers des frais de construction, ont permis un véritable renouvellement du patrimoine immobilier rural. Une nouvelle architecture rurale est en train de naître.

Les fermes construites actuellement dans le Jura central suisse sont toutes des maisons rurales dissociées où la maison d'habitation est séparée des bâtiments d'exploitation (photo no 6).

La maison d'habitation n'a plus guère de caractère rural; les pièces sont aussi nombreuses, aussi spacieuses et aussi claires que celles des appartements urbains de standing moyen. La maison d'habitation de l'agriculteur ne se distingue plus, par son aspect extérieur, d'une villa familiale citadine. Les éléments caractéristiques de la ferme ne doivent par conséquent plus être cherchés dans la maison d'habitation mais uniquement dans les bâtiments d'exploitation et en particulier dans le "rural».

Le type d'étable-grange le plus en faveur actuellement dans le Jura central suisse est celui que nous dénommerons "rural» à éléments juxtaposés en gradins. Le bâtiment est un vaste rez-de-chaussée à tripartition longitudinale. L'orientation parallèle aux courbes de niveau est de règle; la pente, utilisée judicieusement, permet la construction de trois paliers qui constituent l'aire de déchargement des récoltes, le fenil et l'étable. Cette ferme est conçue de façon à limiter les dépenses de temps et d'énergie: les chars de récolte pénètrent dans l'aire de déchargement à l'une des extrémités du palier supérieur, en ressortent à l'autre extrémité sans plan incliné; le déchargement des récoltes se fait par simple déversement sur le deuxième palier, le fenil situé en contrebas; le monte-charge indispensable dans les fermes maltournées est superflu dans le cas de la ferme en 


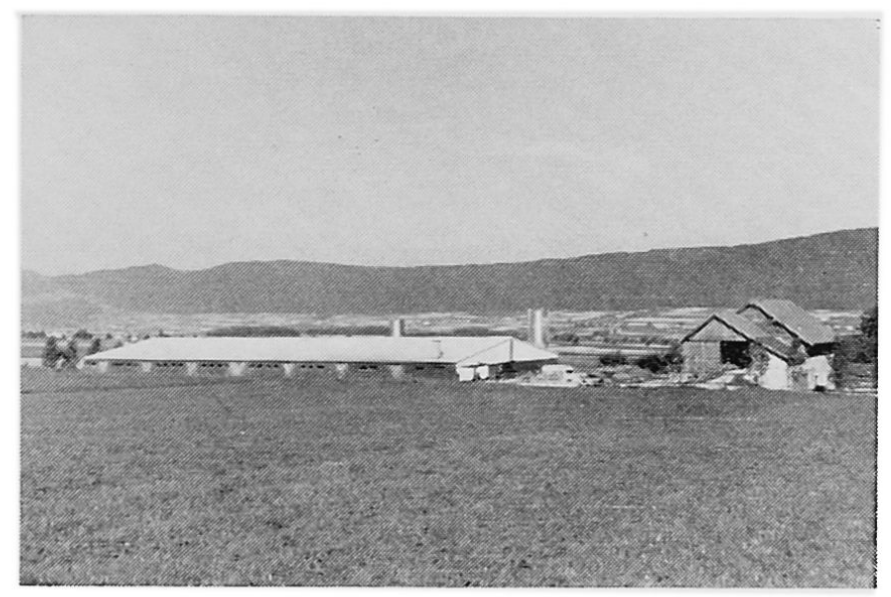

Photo 5. Halle d'élevage de porcs à Boudevilliers

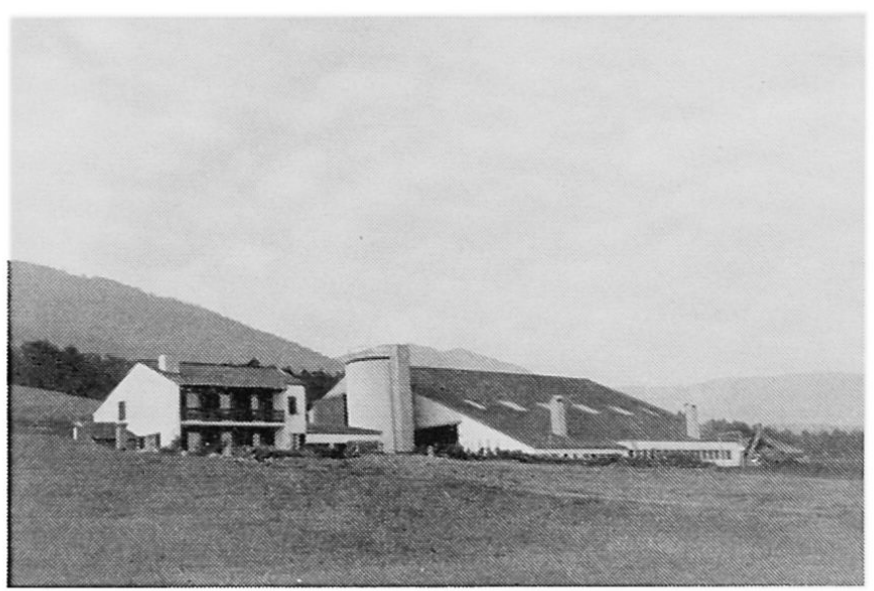

Photo 6. Ferme de «colonisation» à Bevaix. Maison dissociée: maison d'habitation, silo, "rural» à tripartition longitudinale en gradins (aire d'engrangement, grange, étable)

Nous avons vu que le remembrement était la cause principale du renouvellement de la maison rurale; il est également un facteur essentiel de modification du mode de répartition de l'habitat rural. En effet les remembrements permettent fréquemment à quelques agriculteurs trop à l'étroit au village de s'installer sur le pourtour du finage; on assiste à une dispersion de l'habitat rural selon le principe qui veut que la ferme soit au milieu des terres. Certains villages urbanisés ne comptent plus aucune ferme, les agriculteurs étant installés dans la zone agricole périphérique. Il est ainsi possible, grâce aux remembrements, d'effectuer un aménagement régional distinguant zones d'habitation, zones industrielles et zones agricoles. Véritable opération de restructuration foncière, le remembrement permet de remettre un peu d'ordre dans l'utilisation anarchique du sol. En l'absence d'une législation foncière adéquate, le remembrement constitue même le moyen le plus sûr de garantir le maintien des zones agricoles, face à une urbanisation envahissante. Selon la législation suisse, l'affectation des terres agricoles remembrées ne peut en effet pas être modifiée dans les vingt ans qui suivent le remembrement. Les finages remembrés et dans lesquels de nouvelles fermes ont été construites constituent les zones agricoles les plus durables.

L'habitat rural du Jura suisse est soumis actuellement à de fortes influences extra-agricoles et extrarégionales qui modifient la forme de la maison rurale de même que la répartition des fermes; l'habitat rural ne peut par conséquent plus être étudié isolément, comme un élément de folklore, mais bien plutôt en tenant compte des influences économiques, agronomiques, urbaines, parfois extranationales sur l'architecture rurale comme sur le mode de répartition des fermes. 